Journal of Engineering and Applied Sciences 14 (8): 2588-2593, 2019

ISSN: 1816-949X

(C) Medwell Journals, 2019

\title{
Spectrum Allocation in Cognitive Radio Networks Using Genetic Algorithm
}

\author{
Kishore V. Krishnan, A. Bagubali, M. Shanmugasundaram, K. Saravanan and S. Subha Bharathi \\ Vellore Institute of Technology, Vellore, India
}

\begin{abstract}
In this study, we present a method in which the dynamic spectrum gets optimized for its usage in cognitive radio networks. Firstly, based on the interference temperature model, we determine the interference constraints and there is a check on the transmission power of the secondary users. We then formulate the SINR by considering the noise spectral density of the environment and the transmission powers of the surrounding neighbors. Using the Shannon formula, we calculate the co-channel interference between potential links on each channel. Next, using Genetic algorithm, we formulate the spectrum assignment problem. Genetic algorithms are adaptive heuristic search algorithm based on previous and historic results obtained earlier. Although, random, Genetic algorithms exploit historical information to direct the search into a set of better solutions. It is observed that the total network capacity is improved by a significant margin bio inspired algorithms have always been promising in giving optimum solutions.
\end{abstract}

Key words: Spectrum allocation, cognitive radio, Genetic algorithm, direct, network, solutions

\section{INTRODUCTION}

THISA considerable amount of algorithms have been introduced in the domain of spectrum access in cognitive radio networks. These methods have risen by the arrival of $\mathrm{CR}$ technology and change from the usual fixed spectrum assignment to the newer spectrum access techniques (Akyildiz et al., 2006). These models aim at improving the spectrum efficiency by regarding opportunistic and dynamic spectrum access for the secondary users. Considering cognition and reconfigurability as two of the main features and capabilities of the CR node, it comprises mostly two types of phases. The first phase revolves around by having the spectral environment sensed.

The CR user captures the information of the spectrum bands and based on quality level, classify the channels available. The second phase comprises an algorithm of spectrum allocation which apart from satisfying the interference constraints, allows available channels to SU's in such a way that the spectrum efficiency is maximized (Yousefvand et al., 2012). They rely on unrealistic thought that all channels have homogeneous nature with regard to their QoS parameters. It was noted that using a smaller number of simultaneous communication links around the SU's but applying a higher rate on each would lead to a higher total capacity. The heterogeneity of links have been considered in a 3 step manner by computing the maximum allowable power received from receiving nodes and the actual transmission power at transmitting nodes, spectrum between SU's is classified to high and low power transmissions. Basically, the channels have been classified into two categories according to their transmission power and haven't captured the exact link capacity. Models based on interference range have also been used but are not accurate enough though. These models usually define an interference range in the surroundings of the receiver where no transmission is allowed. The conservative nature of these models might degrade the spectrum utilization methods. New link capacity aware algorithms are used to maximize the capacity to such an extent that it includes different QOS parameters like their SNR and bandwidth to consideration along with temperature interference models.

Using the interference graph, the modeling of all co channel interference among the links on various channels takes place. Following that the allocation is formulated in the form of BILP to get a usable set of active links among other links in the graph in such a way that maximum capacity is attained. To tackle the high complexity of these fields, we use radix tree structure in which we have scattered parts of the solution space removed. Following that the problem is formulated onto a radix tree search the non-interfering links in the graph.

Over the past two decades, there has been a rapid increase in the use of wireless applications which in return leads to the need of more bandwidth. Unfortunately, that situation has led to a scarcity in the spectrum which arises due to the inefficient use of EM spectrum. EM spectrum is the electromagnetic radio emitted from different sources which accompany its own frequency and wavelengths. The FCC (Federal Communications Commission) controls

Corresponding Author: Kishore V. Krishnan, Vellore Institute of Technology, Vellore, India 
the EM spectrum and only licensed users can use the EM spectrum. The use of these spectrums varies from locality to locality. The misuse often leads to the efficiency decreasing throughout the spectrum amongst the wireless networks. Hence, cognitive radio has been introduced to take care of these spectrum allocation problems. It is a kind of smart radio which can adapt and change its behavior according to the environment. There can also be a borrowing of unused spectrum from the primary user to a secondary user. Although, this kind of an arrangement must make sure that the primary user's communication is not interrupted. Quite a few issues can arise while the spectrum is handed over from one user to another. The device must be intelligent to sense any holes in the radio environment. It must be able to understand the requirements of application. Studies have proven that the QOS constraints aid in giving efficient wireless communication. The proper sharing under the basis of agreement of the unlicensed spectrum with the licensing authorities should be met with to use spectrum without harming the licensed users. It should also be self-aware of its own operational capabilities.

\section{MATERIALS AND METHODS}

\section{Problem description and goals}

Selecting a proper interference model: The primary step in building a CRN is to choose an appropriate interference model (Kopka and Daly, 1999). The foremost thing in constructing an interference model is to build a channel propagation model relative to the radio environment. We shall consider deterministic path loss, large scale and small scale fading which are the propagation effects in channel models. Under the assumption that there aren't any obstacles in the radio environment, we ignore the fading effects and consider only deterministic path loss effect in our channel model. The next stage is to establish an appropriate transmission channel model which exhibits the effect of interference on the received signal at the receiver end. Primitive channel model were derived from collision channel model where if two or more transmitters try to send a signal to a receiver at the same time. All of the signals would be summed to zero due to the collision effect.

In the recent new era of models, capture channel model is proposed where if one of the received signals is strong enough compared to the other signals (Hill, 1999; Yousefvand et al., 2015) that signal would win over the other and reach the receiver. Vulnerability circle capture model and power capture model belong to the above category. In the vulnerability circle capture model for an

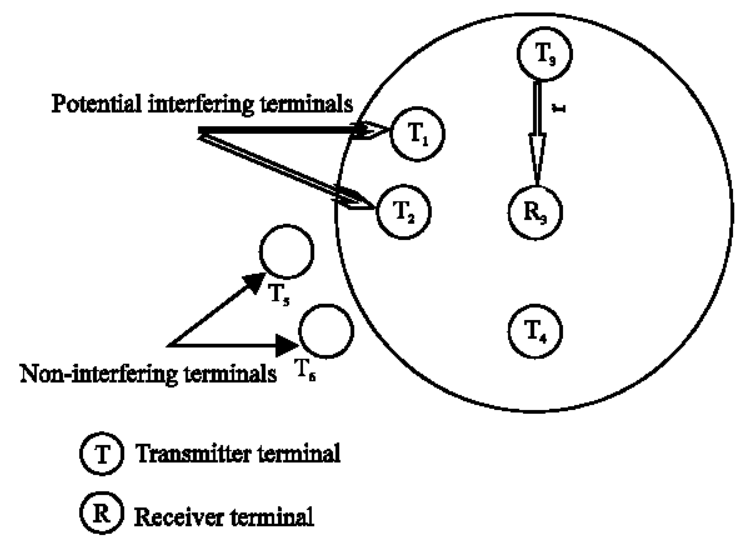

Fig. 1: Vulnerability circle

ith transmitter signal to be successfully received, its receiver power must be more than the power of any other received signal by a factor $\beta$ (Eq. 1):

$$
\frac{p_{r, i}}{p_{r, j}}>\beta \text { for } \rightarrow j=1,2, \ldots, n, j \neq i
$$

Let us consider a model where there is uniform transmit power level for all the transmitters. Mathematically, according to the definition of vulnerability circle model, the radius of the circle is $r=\beta^{1 / n}$

Figure 1 shows the vulnerability circle diagram is demonstrated. The transmitted signal from $T_{1}$ is intended to be Received by $R_{1}$ but it is interfered by the signals transmitted by other transmitters within the vulnerability circle. This is a primitive model which imposes huge restrictions on the transmitted terminals located around the receiver and results in degradation of spectrum efficiency.

For example, if we take a transmitter node $T_{3}$, it's signal is intended to the receiver $\mathrm{R}_{3}$, without causing any interference on any other receiver. In present times, according to practical power capture models, a signal is set to be received successfully at the receiver end if the received power supersedes the power of the accumulated signals from all other receivers by a factor $\beta_{\mathrm{p}}$. Mathematically, we can deduce signal to interference ratio as (Gupta and Kumar, 2000; Ma and Tsang, 2007) (Eq. 2):

$$
\mathrm{SIR}=\frac{\mathrm{p}_{\mathrm{r}, \mathrm{m}}}{\sum_{\mathrm{i} \neq \mathrm{m}} \mathrm{p}_{\mathrm{r}, \mathrm{i}}}>\beta_{\mathrm{p}}
$$

In Eq. 2, the received power at the receiver end of the transmitted signal $\mathrm{i}$ is $\mathrm{p}_{\mathrm{r}, \mathrm{i}}$. In a more realistic scenario, we need to consider additive noise power which is denoted as $\sigma_{2}$. Mathematically, the signal to noise interference ratio (Eq. 3): 


$$
\operatorname{SINR}=\frac{\mathrm{p}_{\mathrm{t}, \mathrm{m}}\left|\mathrm{x}_{\mathrm{m}}-\mathrm{x}_{\mathrm{R}(\mathrm{m})}\right|^{2}}{\sigma^{2}+\sum_{\mathrm{t} \neq \mathrm{m}, \mathrm{n} \in \mathrm{N}} \mathrm{p}_{\mathrm{t}, \mathrm{m}}\left|\mathrm{x}_{\mathrm{m}}-\mathrm{x}_{\mathrm{R}(\mathrm{m})}\right|^{2}}>\beta_{\mathrm{p}}
$$

In Eq. 3, $\left|\mathrm{x}_{\mathrm{m}}-\mathrm{x}_{\mathrm{R}(\mathrm{m})}\right|$ implies the distance between transmitter $m$ and its receiver. The bandwidth in the above model is at a constant rate $W_{m}$, for a given link $\left(T_{m}, R_{m}\right)$. For reliable transmission to take place at radio environment, the SINR for any given link should exceed a threshold. Otherwise, it is nullified. From the definition of Shannon's formula for link capacity, the data rate for the given link $\left(\mathrm{T}_{\mathrm{m}}, \mathrm{R}_{\mathrm{m}}\right)$ (Eq. 4):

$$
\mathrm{W}_{\mathrm{m}}=\mathrm{B} \log \left(1+\frac{\mathrm{p}_{\mathrm{t}, \mathrm{m}}\left|\mathrm{x}_{\mathrm{m}}-\mathrm{x}_{\mathrm{R}(\mathrm{m})}\right|^{2}}{\sigma^{2}+\sum_{\mathrm{t} \neq \mathrm{m}, \mathrm{n} \in \mathrm{N}} \mathrm{p}_{\mathrm{t}, \mathrm{m}}\left|\mathrm{x}_{\mathrm{m}}-\mathrm{x}_{\mathrm{R}(\mathrm{m})}\right|^{2}}\right)
$$

$B$ represents channel bandwidth and $\sigma_{2}=N_{0} / 2$ denotes noise spectral density.

Genetic algorithm: Evolutionary algorithm was found to be promising in solving the optimization problem. Hence, we have opted for Genetic algorithm to maximize the network capacity, thus, optimizing the spectrum as a whole.

Approach: The algorithm is carried out by random choice of chromosomes that have a particular trait which are computed through generations. The fitness of a given chromosome at a particular generation is evaluated on the basis of a random probabilistic calculation. Calculation of fitness of chromosomes is based on individuality, the process continues for several generations iteratively till we reach an optimum solution.

Initialization: $\mathrm{N}$ chromosomes re generated randomly which form the initial population. Probable solutions for the given problem are part of the population stated above.

Fitness measure: This stage includes computing the fitness of chromosomes which constitute the initial population.

\section{Construction of new population}

Selection: A set of chromosomes is selected based on the criteria of fitness from the available population.

Crossover: New progeny are part of the upcoming generation by the method of crossover. Progeny are formed or reproduced based on the probability of crossover.
Mutation: The chromosome structure of the offspring is converted from string to binary format and the binary values are toggled at a particular point to retain the diversity of the upcoming generations.

Stopping criteria: This iterative method is run through multiple times till we reach an optimum solution. It is also found that the stopping criteria are relative to the complexity of the chromosome structure.

System model: Let us assume that there are $\mathrm{K}$ primary users in the spectral environment transmitting to each other through $\mathrm{C}$ channels and $\mathrm{L}$ secondary users. Competing against each other to access the spectrum opportunistically. Let us define a matrix to determine the availability of link between two users. Mathematically (Eq. 5):

$$
\mathrm{B}_{\mathrm{c}}=\mathrm{b}_{\mathrm{m}, \mathrm{n}, \mathrm{c}} \mid \mathrm{b}_{\mathrm{m}, \mathrm{n}, \propto\{\{0,1\}}
$$

In the above equation $\mathrm{b}_{\mathrm{m}, \mathrm{n}, \mathrm{c}}=1$ if both secondary users $m$ and $n$ sense the channel $\mathrm{c}$ as a free channel. If not $b_{m, n, c}=0$. In order to infer if the channel $c$ is free or not each secondary user relates it's observed interference on that channel with the interference temperature threshold. In fact, mathematically, secondary user $m$ senses the channel free if the following condition is satisfied (Eq. 6):

$$
\sum_{\mathrm{k}=1, \mathrm{k} \neq \mathrm{i}}^{\mathrm{M}} \operatorname{Pr}_{\mathrm{m}, \mathrm{n}, \mathrm{c}}+\mathrm{N}_{\mathrm{c}} \leq \mathrm{T}_{\mathrm{c}}
$$

In Eq. 6, $\operatorname{Pr}_{\mathrm{m}, \mathrm{n}, \mathrm{c}}$ is the power received by secondary user $m$ from a signal sent by $k$ th primary user via. channel c. The noise on the channel $\mathrm{c}$ is given by $\mathrm{kth}$. Soon after discovering a set of potential links for each of the users, each link is associated by a weight. Since, out motto is to increase the network capacity, we allot each link as link capacity. Each secondary user $m$, raises its transmission power for a given channel c by simultaneously being in accordance with the interference constraints of the primary users. In fact, mathematically, we can strike a relation for the transmission power of the secondary user by determining the maximum allowable transmission power. If the transmission power from the secondary user exceeds the limit by a factor $\lambda$, then, the condition on interference threshold is violate. Hence we canmathematically deduce the following Eq. 7 and 8:

$$
\mathrm{P}_{\mathrm{m}, \mathrm{c}} \leq \operatorname{Pmax}_{\mathrm{m}, \mathrm{c}} \forall \mathrm{r}: \mathrm{T}_{\mathrm{c}, 1} \leq \mathrm{r} \leq \mathrm{M}
$$




$$
\mathrm{P}_{\mathrm{m}, \mathrm{c}}=\left(\mathrm{Pmax}_{\mathrm{m}, \mathrm{c}}+\lambda\right) \ni \mathrm{r}: \mathrm{T}_{\mathrm{r}, \mathrm{c}}>\mathrm{T}, \mathrm{c} \lambda>0
$$

Apart from the maximum limit imposed on the power transmitted by the secondary user, there is also a minimum transmission power level required for each secondary user's transmission, so that, the condition of minimum SINR is met. Each secondary user has to choose its transmission power from its minimum and maximum bounds. Mathematically, we can deduce the relation as $\mathrm{P} \in\left(\mathrm{Pmin}_{\mathrm{m},}, \mathrm{Pmax}_{\mathrm{m}, c}\right)$. Having deduced the transmission power of each secondary user $m$ on their channels, we shall determine link capacity of each potential links on these channels. We shall construct the weight matrix $\mathrm{W}_{\mathrm{c}}$ as $\mathrm{w}_{\mathrm{m}, \mathrm{n}, \mathrm{c}}$. Each element in this matrix represents weight of the link in between transmitter $\mathrm{m}$ and receiver $\mathrm{n}$ on channel c mathematically, we can determine it as Eq. 9:

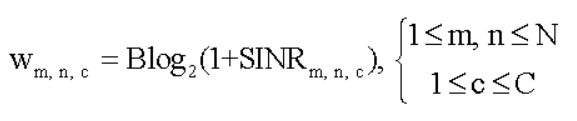

In the above equation, $\mathrm{B}$ implies bandwidth on channel $\mathrm{c}$ and SINR $\mathrm{m}_{\mathrm{m}}$ implies the SINR of the link amidst transmitter $\mathrm{m}$ and receiver $\mathrm{n}$ on channel $\mathrm{c}$, we can calculate SINR of the potential links as Eq. 10:

$$
\mathrm{SINR}_{\mathrm{m}, \mathrm{n}, \mathrm{c}}=\frac{\mathrm{Pr}_{\mathrm{n}, \mathrm{m}, \mathrm{c}}}{\mathrm{N}+\sum_{1=1,1 \neq \mathrm{m}}^{\mathrm{K}} \operatorname{Pr}_{\mathrm{m}, 1, \mathrm{c}}}
$$

In the above equation, the power received by receiver $\mathrm{n}$ is $\mathrm{Pr}_{\mathrm{n}, \mathrm{m}, \mathrm{c}}$ which is from transmitter $\mathrm{m}$ on channel $\mathrm{c}$. The background noise and the channel are denoted by $\mathrm{n}$. The total number of active transmitters on channel $\mathrm{c}$ is $\mathrm{k}$. In order to control the co channel interference on each of the potential links, we construct a matrix to remove all possible conflicts (Eq. 11):

$$
\mathrm{G}_{\mathrm{c}}=\mathrm{G}_{\mathrm{c}_{\mathrm{m}, \mathrm{p}, \mathrm{p}, \mathrm{q}}} \in\{0,1\}
$$

In the above equation, $\mathrm{G}_{\mathrm{c}_{\mathrm{m}, \ldots, 4}}$ will be taken as unity under the condition that a potential link between transmitter $m$ and receiver $n$ intervenes with the link between transmitter $\mathrm{p}$ and receiver $\mathrm{q}$ on channel $\mathrm{c}$ if not, $\mathrm{G}_{\mathrm{c}_{\mathrm{m} 2 \mathrm{x}, \mathrm{q}}}$ will be nullified. One link intervenes with the other, under the condition that coinciding transmissions on these two links result in the SINR of one of them to dip below a certain threshold SINR required to have successful transmission. To avoid non-active links to be part of our solution set, we construct a channel assignment matrix which remove non-active links. This matrix consists of binary values $(0,1)$ (Eq. 12):

$$
\mathrm{D}_{\mathrm{c}}=\mathrm{d}_{\mathrm{m}, \mathrm{n}, \mathrm{c}} \in\{0,1\}
$$

Thus, it can be said that, when $\mathrm{d}_{\mathrm{m}, \mathrm{n}}=1$, then, the link between transmitter $m$ and receiver $n$ will be part of a bunch of potential links for the channel $\mathrm{c}$ if not $\mathrm{d}_{\mathrm{m}, \mathrm{n}, \mathrm{c}}$ will be nullified. In order to put a check on link conflicts, we impose the following condition on the spectral environment (Eq. 13):

$$
a_{m, n, c}+a_{p, q, c} \leq \text { if }\left\{\begin{array}{c}
G_{c_{m, n, p, q}}=1 \\
1 \leq m, n, p, q \leq N
\end{array}\right.
$$

We can determine the total network capacity of the spectral environment by taking the sum of products of channel assignment matrix and link capacity matrix. Mathematically, Eq. 14:

$$
\mathrm{T}=\operatorname{Agg}\left(\sum_{\mathrm{c}=1}^{\mathrm{c}} \mathrm{D}_{\mathrm{c}} \mathrm{W}_{\mathrm{c}}\right)
$$

In Eq. 14, Agg is the operator that calculates the addition of all entries of the matrix. $\mathrm{T}$ indicates the overall network capacity of the spectral environment. It is observed that $D_{c}$ and $W_{c}$ have uniform dimension. The above equation can be made as an optimized equation which can be usedas an objective function in an evolutionary algorithm such as Genetic algorithm.

\section{RESULTS AND DISCUSSION}

We have built an environmental setup comprising of 4 primary users and 16 secondary users which are spreadacross an area of 1024, square meters as shown in Fig. 2. As discussed earlier, we shall operate the cognition cyclestages to this spectral environment. With a supposition that secondary users operate through six operational channels. We have restricted interference on each of the channels byimposing an interference temperature limit of $10 \mathrm{~dB}$ on eachof the channels. Also, we have a requirement of at least unity as the SINR.

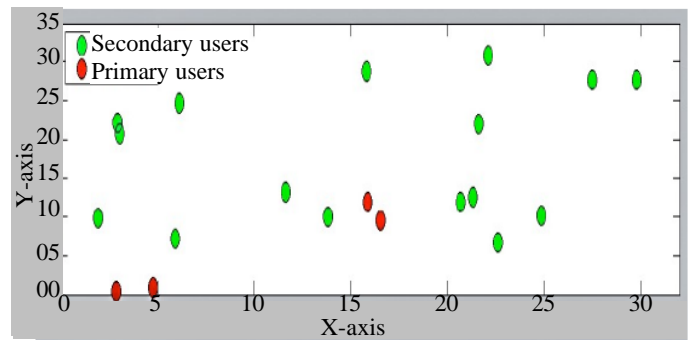

Fig. 2: Spatial distribution of PUs and SUs 


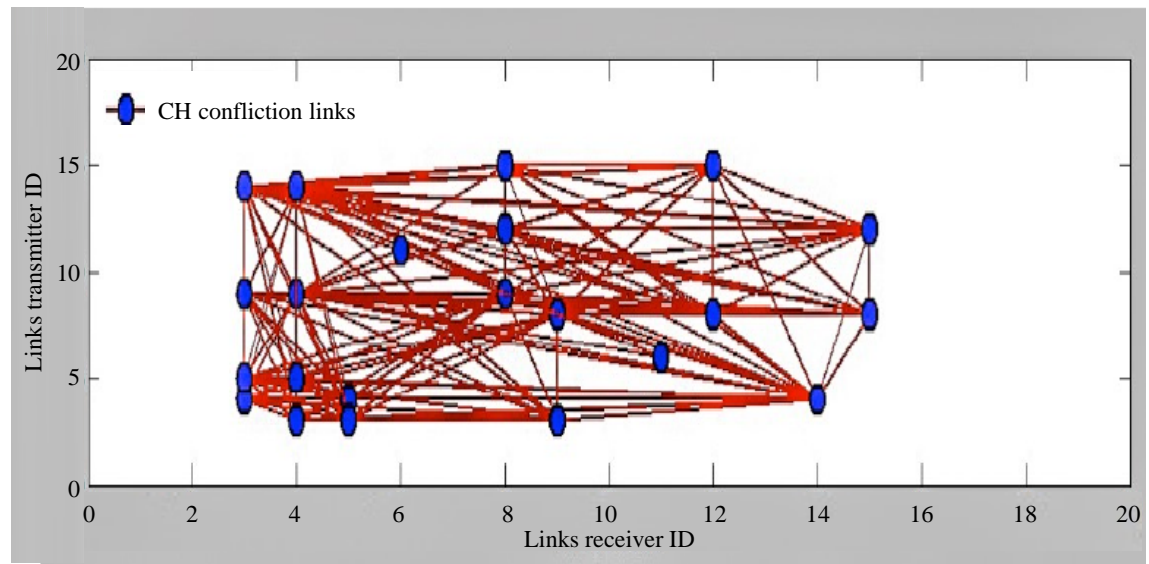

Fig. 3: Interference graph for channel 1

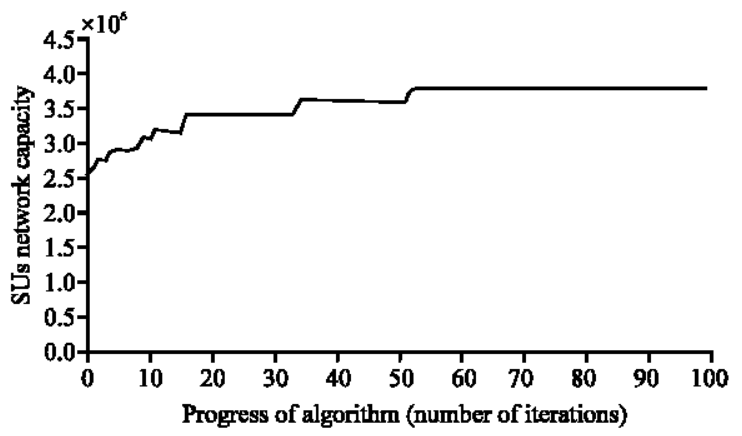

Fig. 4: Total network capacity of the secondary users (scenario-1) (Genetic algorithm)

For trustworthy transmission, we initially spot the potential links and determine their network capacities and SINRs. We neglect the links with SINR $<1$. We build aninterference graph for each channel as shown in Fig. 3 which represents the interference graph of channel 1. It is observed that Genetic algorithm converges to an optimum solution as the number of iterations increases as shown in Fig. 4.

We take another case where we have 25 secondary users and 5 primary users, spatially distributed across 2500 squaremeters. It has been observed that in this scenario that by increasing the number of active links among the secondary users, it need not certainly improve the network capacity by a significant factor as shown in Fig. 5. The network capacity may increase only if, we consider all the links as uniform whichis not practical. Intrinsic trait of Genetic algorithm is highly arbitrary due to processes like crossover and mutation.

The progression of iterations in GA does not mandate an optimum solution which is evident from the periodic movement in the coming generations. In our proposed model, we have distributed the nodes in random fashion due to which secondary users can control the transmission power.

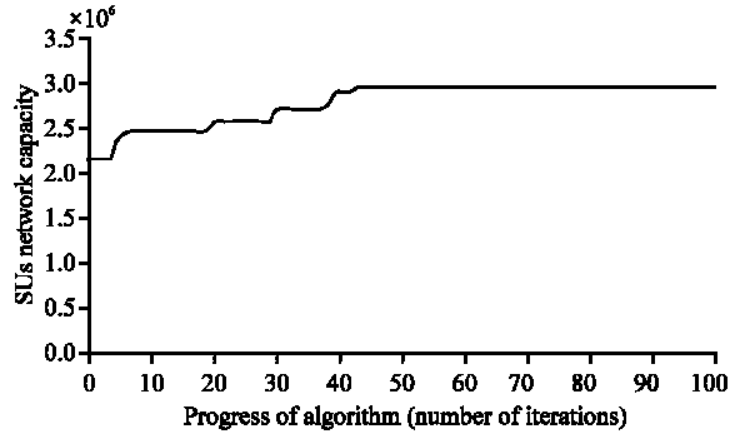

Fig. 5: Total network capacity of secondary users (scenario-2) (Genetic algorithm)

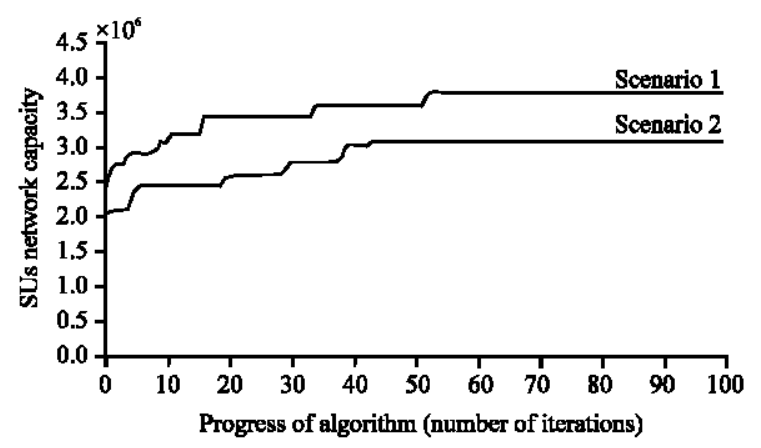

Fig. 6: Overall network capacity (Genetic algorithm)

Decreasing the distance between the secondary users in populated areas which in turn increases the link SINR. Also, does this there is more number of links that satisfy the SINR criteria. Therefore, improving the overall network capacity significantly as shown in Fig. 6.

\section{CONCLUSION}

By providing opportunistic access to usable and available channels for the secondary users, the 
technology of cognitive radio proves itself to be highly promising. And in that matter highly helps in making the usage of spectrum better. By optimization of variable number of parameters, the enhancing of spectrum is met with and conducted. In this study, we have come up with little interference limited capacity known spectrum allowed design, to facilitate maximum capacity in cognitive radio. The results shown in the simulation prove that it is able to achieve higher network capacity by inculcating the suggested model than the normal methods used for allocating the spectrum wisely. It has also come to our observation that by maximizing the number of links that are active amongst the secondary users, it need not directly lead to the increase or maximization of the capacity of the network. It could be taken like that if, we make an assumption that all the links are equal. But that form of an assumption would not be pragmatic to the approach of our scenario. We have also noticed that the scenarios in which the secondary users are equipped with the capability to control power, it is able to achieve higher capacity given that the secondary users are distributed in a random manner as compared to the case in which they are laid out in a definite manner.

\section{REFERENCES}

Akyildiz, I.F., W.Y. Lee, M.C. Vuran and S. Mohanty, 2006. Next generation/dynamic spectrum access/cognitive radio wireless networks: A survey. Comput. Networks, 50: 2127-2159.
Gupta, P. and P.R. Kumar, 2000. The capacity of wireless networks. IEEE Trans. Inform. Theory, 46: 388-404.

Hill, R.R., 1999. A monte-carlo study of genetic algorithm initial population generation methods. Proceedings of the 31st International Conference on Winter simulation: Simulation-A bridge to the future Vol. 1, December 5-8, 1999, ACM, Phoenix, Arizona, USA., pp: $543-547$

Kopka, H. and P.W. Daly, 1999. A Guide to LATEX: Document Preparation for Beginners and Advanced Users. 3rd Edn., Addison-Wesley, Boston, Massachusetts, USA., ISBN:9780201398250, Pages: 600.

Ma, M. and D.H. Tsang, 2007. Efficient spectrum sharing and power control in cognitive radio networks. Proceedings of the 1st International Workshop on Cognitive Wireless Networks, August 14-17, 2007 , ACM, New York, USA., ISBN:978-1-60558-868-1, pp: $1-7$.

Yousefvand, M., N. Ansari and S. Khorsandi, 2015. Maximizing network capacity of cognitive radio networks by capacity-aware spectrum allocation. IEEE. Trans. Wireless Commu., 14: 5058-5067.

Yousefvand, M., S. Khorsandi and A. Mohammadi, 2012. Interference-constraint spectrum allocation model for cognitive radio networks. Proceedings of the 20126 th IEEE International Conference on Intelligent Systems (IS), September 6-8, 2012, IEEE, Sofia, Bulgaria, ISBN:978-1-4673-2276-8, pp: 357-362. 